\title{
Evaluating the Function of Hippocampal Subregions With High-Resolution MRI in Alzheimer's Disease and Aging
}

\author{
SCOTT A. SMALL, ${ }^{1,3 *}$ ARUN S. NAVA, ${ }^{1,3}$ GERARD M. PERERA, ${ }^{2}$ ROBERT DELAPAZ, ${ }^{2}$ AND YAAKOV STERN ${ }^{1,3}$ \\ ${ }^{1}$ Department of Neurology, Columbia University College of Physicians and Surgeons, New York, New York 10032 \\ ${ }^{2}$ Department of Radiology, Columbia University College of Physicians and Surgeons, New York, New York 10032 \\ ${ }^{3}$ Taub Center for Alzheimer's Disease Research in the City of New York, Columbia University College of Physicians and Surgeons, \\ New York, New York 10032
}

\author{
KEY WORDS memory; memory decline; aging; Alzheimer's Disease; fMRI
}

\begin{abstract}
Memory ability declines in older age groups. There is a growing list of physiological processes that target the hippocampal formation in an age-related fashion, and some might underlie the hippocampal component of memory decline. The hippocampal formation is comprised of separate subregions, and physiological processes differentially target these subregions. The ability to evaluate the functional integrity of individual subregions-performing subregional analysis-is a major clinical goal since it can aid in the diagnosis of memory decline, as well as in elucidating mechanisms of disease and testing potential interventions. Because of its superior spatial resolution, magnetic resonance imaging (MRI) is best suited to accomplish this goal. Despite limited success, most functional MRI (fMRI) protocols have difficulty in performing complete subregional analysis of the hippocampal formation. Here we address sources of difficulty by (1) generating T2* -weighted maps of the hippocampal formation with sub-millimeter resolution; and (2) by adapting an approach used by animal investigators to identify the hippocampal subregions using anatomical landmarks. The protocol is tested in patients with Alzheimer's disease and in healthy controls, in an effort to determine whether it can detect neuronal dysfunction. Results showed diminished signal in the hippocampal formation of patients with Alzheimer's disease (AD) compared to controls, and multivariate analysis showed that this difference was most prominent in the entorhinal cortex. The protocol can be used to perform subregional analysis of the hippocampal formation. Testing the protocol in other clinical populations is needed to demonstrate its efficacy in evaluating the neuronal integrity of all hippocampal subregions. Microsc. Res. Tech. 51:101-108, 2000. ๑ 2000 Wiley-Liss, Inc.
\end{abstract}

\section{INTRODUCTION}

Memory loss is one of the most common complaints among healthy elderly, and a number of cross-sectional as well as longitudinal studies have documented that memory performance declines with age (Petersen et al., 1992; Small et al., 1999b). Episodic memories represent one memory subsystem sensitive to age-related effects (Craik et al., 1995). The consolidation, storage, and retrieval of episodic memories require a complex interplay among multiple cortical sites (Zola-Morgan and Squire, 1993). Evidence has implicated the hippocampal formation as one, but not necessarily the only, locus of dysfunction associated with age-related memory decline (Barnes, 1994; Geinisman et al., 1995).

There is an ever-growing list of processes that target the hippocampal formation in an age-related fashion, and some might underlie the hippocampal component of memory decline. This list includes Alzheimer's disease, which results in isolated memory impairment early in its course (Jacobs et al., 1995), age-dependent changes in hormonal blood levels and cerebrovascular supply (de la Torre et al., 1992; McEwen 1994), and age-dependent accrual of oxidative stress (Forster et al., 1996). Hippocampal dysfunction can also occur in the absence of a clear etiology, which might reflect time-dependant limitations inherent to neuronal phys- iology and/or longevity (Gazzaley et al., 1996). Recent studies are beginning to identify genetic risk factors for age-related memory decline, which could account for individual variability in memory decline (Helkala et al., 1995).

The hippocampal formation is not a unitary structure, but rather, it is comprised of separate anatomical subregions: the entorhinal cortex, the subiculum, the CA subfields, and the dentate gyrus (Amaral and Witter 1989). The neuronal populations within each subregion, expressing unique electrophysiological and molecular phenotypes (Nicoll and Malenka 1995), are differentially vulnerable to mechanisms of dysfunction. For example, the entorhinal cortex is the first subregion targeted by Alzheimer's pathology (Braak and Braak 1996), the subiculum undergoes time-dependent cell death, the CA1 subregion is most sensitive to cerebrovascular changes (Simic et al., 1997; West, 1993),

*Correspondence to: Scott A. Small, Columbia University, College of Physicians and Surgeons, PH \#19, 630 West 168 Street, New York, NY 10032.

E-mail: sas68@columbia.edu

This work was supported in part by federal grants AG08702, AG00949, AG07232; the Beeson Faculty Scholar Award from the American Federation of Aging; by the Charles S. Robertson gift from the Banbary Fund.

Received 1 March 2000; accepted in revised form 5 June 2000 
and the dentate gyrus is most sensitive to changes in testosterone levels (Galea et al., 1999).

Using currently available neuropsychological tests and imaging methods, it is possible to localize a memory deficit to the hippocampal formation. These techniques, however, have difficulty in resolving a memory deficit to specific hippocampal subregions. Because the hippocampal subregions are interconnected to form a circuit, injuring any subregion can be equipotent in impairing global hippocampal function. The ability to evaluate the functional integrity of individual hippocampal subregions-performing subregional analysis-is a major clinical goal since it can aid in the diagnosis of memory decline in otherwise healthy elderly, as well as in elucidating mechanisms of disease and testing potential interventions.

Among functional imaging techniques, functional magnetic resonance imaging (fMRI) possesses the greatest spatial resolution. We and others have shown that fMRI can be developed to assess some (Gabrieli et al., 1997; Small et al., 1999), but not all, hippocampal subregions. These protocols have difficulty in performing a more complete subregional analysis of the hippocampus. The millimeter resolution provided by most fMRI protocols is simply not good enough to reliably assess the smaller hippocampal subregions with dimensions of $2-3 \mathrm{~mm}$. Volumetric measures of the hippocampal formation and/or the entorhinal cortex have also been used successfully to distinguish Alzheimer's patients, mild cognitive impairment, and healthy elderly (Bobinski et al., 1999; Golomb et al., 1994).

Functional MRI is based on the principle that a T2* (magnetic susceptibility) signal is sensitive to shifts in oxyhemoglobin/deoxyhemoglobin ratios (Ogawa et al., 1990), and that neuronal metabolism is correlated with this ratio because of hemodynamic coupling. Thus, the $\mathrm{T} 2 *$ signal is an indirect measure of neuronal function. Since it was first developed in the early 1990 s, nearly all fMRI studies have examined the dynamic shift in $\mathrm{T} 2 *$ signal associated with behavioral activation tasks. Most fMRI studies have been designed to establish the functional neuroanatomy of various cognitive processes within a normal population. For these goals, a withingroup design comparing signal intensity between "activation" and "baseline" tasks has proven extremely successful, and has led to the remarkable explosion of the use of fMRI in cognitive neuroscience.

Clinical questions about brain function are inherently different in that they require a between-group design. Dynamic fMRI studies can be used to demonstrate differences in brain activation between a clinical and a control group. For example, we have shown differences in hippocampal activation associated with visual processing between patients with Alzheimer's disease compared to age-matched controls (Small et al., 1999). Nevertheless, requiring a behavioral activation task requires that a clinical group be able to perform a cognitive activation task. In our dynamic fMRI studies we were only able to evaluate patients with mild disease, because patients with more profound dementia could not understand the instructions of the activation task. Furthermore, detecting brain activation in association with a behavioral task is most readily accomplished using rapid acquisition pulse sequences sensitive to dynamic shifts in $\mathrm{T} 2 *$ signal. In most cases, "rapid" MR is acquired using echo-planar imaging techniques, requiring rapid shifting of magnetic gradients, which effectively limits spatial resolution to within the millimeter dimension (Frahm et al., 1993). This might be acceptable for evaluating larger hippocampal subregions, such as the entorhinal cortex, but it is inadequate for smaller subregions. For these subregions, submillimeter resolution is required.

The first imaging studies to establish that $\mathrm{T} 2{ }^{*}$ weighted signals are sensitive to relative concentrations of deoxyhemoglobin did so with "conventional" gradient-echo pulse sequences (Ogawa et al., 1990a,1990b). Although similar pulse sequences are still used by some groups, the vast majority of fMRI studies use echo-planar imaging. Echo-planar imaging allows large brain volumes to be sampled more frequently, a feature attractive for studies that wish to establish a brain network whose dynamic signal changes with an activation task. For clinical purposes, however, the sacrifice of temporal resolution may be offset by the improved spatial resolution afforded by conventional gradient-echo imaging, which can easily achieve the sub-millimeter level desired.

A second difficulty in performing subregional analysis of the hippocampal formation, aside from needing better spatial resolution, is identifying the subregions with MR images (Stern and Hasselmo 1999). This is problematic since delineating the precise border zones among the hippocampal subregions requires histological staining in post-mortem tissue. Animal investigators who study hippocampal electrophysiology in in vitro slices are confronted with a similar problem, since they are prohibited from using staining techniques, yet need to identify the subregions. The approach developed by these investigators relies on identifying the general locale of the hippocampal using visible anatomical landmarks, and sampling data-points from within a subregion (Teyler, 1980). The main landmarks used are the external morphology and the internal white matter tracts of the hippocampal formation. Since these landmarks are optimally visualized in the middle aspect of the hippocampal formation-with greater anatomical complexity in its anterior and posterior aspects - a single transverse slice taken through the body of the hippocampus is selected for analysis.

Here, we use a protocol developed to perform subregional analysis of hippocampal function. First, we trade off temporal for spatial resolution to generate T2* maps of the hippocampal formation with sub-millimeter resolution. Second, we adapt an approach used by animal investigators to identify the hippocampal subregions using anatomical landmarks visualized by MR images. We test this protocol by evaluating the hippocampus in subjects with probable Alzheimer's disease compared to healthy elderly. Because the hippocampal formation is a prominent site of Alzheimer's pathology (Braak and Braak, 1996), we predicted that this MR protocol should dissociate the two groups.

\section{STUDY 1}

The goal of this first study was to offer preliminary evidence that "resting" T2* -weighted signal can be used, in principle, to detect neuronal dysfunction. We analyze data previously acquired in a study that used dynamic echo-planar imaging to investigate healthy 
elderly and patients with Alzheimer's disease (AD). In the prior study we tested for group differences in dynamic $\mathrm{T} 2 *$-weighted signal associated with an activation task; in the current study we test for group differences in resting signal.

\section{Subjects}

Two groups of individuals over the age of 65 participated in the study: Four subjects with normal memory and 5 subjects with Alzheimer's disease.

Subjects for groups were drawn from a single community and were evaluated prospectively with annual medical, neurological, and neuropsychological exams (Stern et al., 1992). All subjects were presented in a consensus conference comprised of neurologists, psychiatrists, and neuropsychologists, and were excluded if at any time point they fulfilled DSM-IV criteria for dementia, or if they were diagnosed with stroke, Parkinson's disease, or depression. Subjects were also excluded if they were diagnosed with "questionable dementia," a category applied if neuropsychological test performance was below cutoff scores established for this community (Stern et al., 1992), but were not extensive enough to fulfill dementia criteria. A slope of memory performance over time was calculated for each subject by performing a linear regression of the total recall score of the Selective Reminding Test (Buschke and Fuld, 1974). Subjects were assigned to the normal memory group if their memory did not decline over time.

Subjects for the $\mathrm{AD}$ group were selected from a clinical setting. They all fulfilled DSM IV criteria for dementia, and NINCDS-ADRD (McKhann et al., 1984) criteria were used for the diagnosis of probable AD. Only subjects with mild dementia [clinical rating scale (Morris, 1993) = 1] were selected based on preliminary studies showing that individuals with more profound dementia could not perform the cognitive activation task.

The healthy elderly (mean age $=80 ;$ S.D $=5.3$ ) were on average older than the AD patients (mean age $=$ 70.5; S.D = 5.5). This difference, however, was not statistically significant.

\section{Task Design}

Stimuli were black-and-white photographs taken from a high-school yearbook. All faces were stored digitally in a Macintosh laptop computer, and were organized using the Psyscope software program. Faces were projected onto a back-projection screen located at the foot of the MR bed via a LCD projector located outside the scanner room. Subjects viewed the screen via a prism system located in the head coil. All faces were presented one at a time, 4.4 seconds per stimulus, with an interstimulus interval of 0.6 seconds.

In order to accommodate the needs of the subjects, some with memory decline and/or dementia, the task was kept simple and relatively brief. The task lasted 4 minutes during which time an activation phase, made up of 12 faces, alternated with a baseline phase consisting of a fixation point. Task design was as follows: Activation phases occurred during the first and third minutes, and baseline phases during the second and fourth minutes. Subjects were instructed to push one button if a face was male and the other button if a face was female. All subjects, including the $\mathrm{AD}$ patients, were able to discriminate gender with $100 \%$ accuracy. This documented that all subjects were successfully engaged in performing the task, a concern particularly relevant for studies where some subjects have dementia. Left or right button responses that corresponded to gender were pseudorandomized across different subjects within each experimental group. Subjects were instructed to remember the faces for future testing.

\section{Scanning Methods}

Scanning was done on a 1.5-tesla magnetic resonance scanner retrofitted for echo planner imaging. A gradient echo sequence [echo time $(\mathrm{TE})=60 \mathrm{~ms}$; repetition time $(\mathrm{TR})=2.5 \mathrm{~s}$; flip angle $\left.=30^{\circ}\right]$ and a standard quadrature head coil were used to acquire $\mathrm{T} 2 *$-weighted images with an inplane resolution of $2.3 \times 2.3 \mathrm{~mm}(64 \times 64$ matrix; $15 \mathrm{~cm}$ field of view $)$. High-resolution "Fast Multiplanar Inversion Recovery" images were also acquired using the same spatial coordinates [Echo time $(\mathrm{TE})=43 \mathrm{~ms}$; repetition time $(\mathrm{TR})=6,500 \mathrm{~ms}$; inversion time $(\mathrm{TI})=200 \mathrm{~ms} ; 512 \times$ 512 matrix; $15 \mathrm{~cm}$ field of view]. Six 5-mm slices were selected that were oriented along the long axis of the hippocampal formation. The choice of the most anterior image was based on identification of the alveus and on the image where the temporal horn resides both laterally and superior to the hippocampus proper. Thus, the most posterior slice was approximately $35 \mathrm{~mm}$ caudal to the amygdala, ensuring incorporation of the entorhinal cortex (Amaral and Insausti 1990).

Image analysis was performed on a Silicon Graphics Indigo II work station, using image display and analysis software packages (MEDx Sensor Systems, IDL Research Systems). The AIR (Woods et al., 1993) program was applied to correct for head motion and to co-register the volumes. Although the short acquisition time of the runs enhanced the goodness-of-fit of the algorithm head, motion is particularly important in this study, as small brain regions were under investigation. Studies were rejected if a shift of greater than $1 \mathrm{~mm}$ over the scanning time period was detected in any direction after co-registration. Spatial filtering was not used in post-acquisition processing because it effectively reduces both spatial resolution and spatial fidelity

Fixed anatomical criteria (Amaral and Insausti, 1990; Duvernoy, 1998) were used to define identify the hippocampal formation, on a slice-by-slice basis. Of the six transverse slices acquired, a single slice was selected that was $20 \mathrm{~mm}$ posterior to the amygdala, which was also anterior to the lateral geniculate nucleus. In order to prevent a potential hemispheric bias, an arbitrary decision was made to analyze the left hippocampus for each subject. A region of interest was drawn around the left hippocampal formation including the entorhinal cortex. Average signal intensity during the baseline phases of the activation task was determined for each subject

\section{Data Analysis}

In order to test for differences in resting $\mathrm{T} 2$ * $_{-}$ weighted signal between experimental groups acquired with echo-planar imaging, an independent-sample $t$ - 


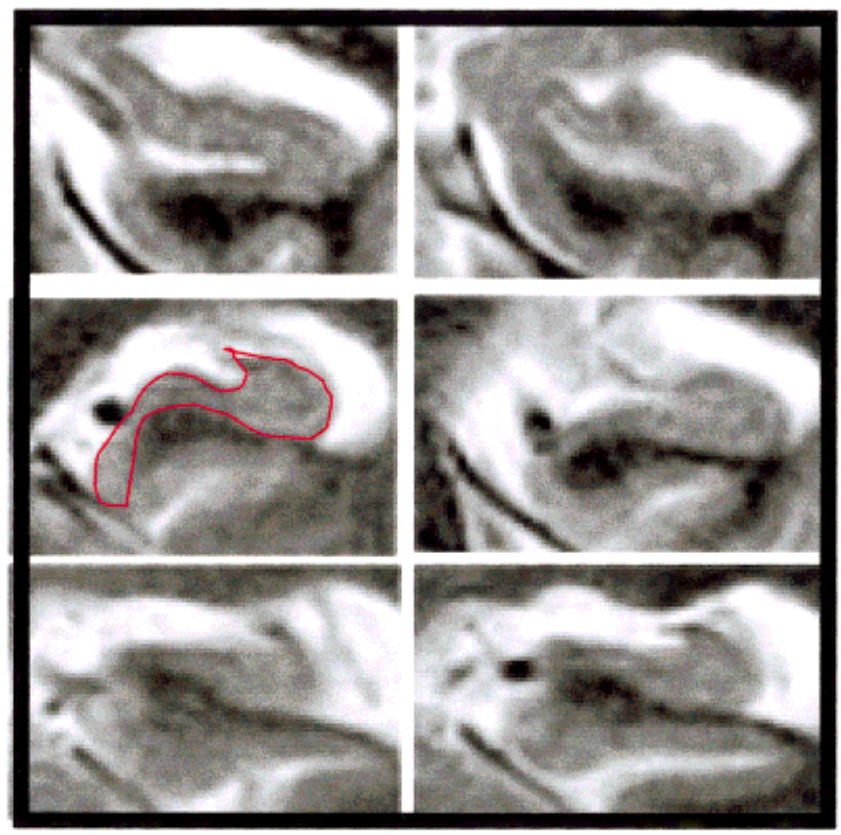

Fig. 1. Identifying the hippocampal formation for echo-planar image analysis. A region of interest, including all subregions of the hippocampal formation, was selected from high-resolution "structural" images. This ROI was used to measure resting T2* -weighted signal from echo-planar images in a group of healthy elderly and a group of patients with Alzheimer's disease.

test was performed. The group variables were patients with Alzheimer's disease and healthy elderly with normal memory, and the test variable was average signal intensity acquired in the hippocampal ROI during the baseline phases of the activation task.

\section{Results}

Independent-sample $t$-tests revealed that $\mathrm{AD}$ patients had significantly lower signal intensity in the resting phases of the echo-planar imaging study (mean for healthy elderly $=151$, S.D. $=18.8$; mean for $\mathrm{AD}=$ 91. S.D. $=24.2)$, $\mathrm{t}(7)=4.0, P<.01$.

\section{Discussion}

A few studies have used "resting" T2* signal acquired with rapid echo-planar imaging, to map brain function within a normal population (Biswal et al., 1995; Xiong et al., 1999). Here, we show that "resting" $\mathrm{T} 2 *$ signal is lower in the hippocampal formation of a group of patient with $\mathrm{AD}$ compared to healthy elderly. In the general discussion below, we discuss proposed mechanisms for this diminished signal.

Although we used the baseline phases as a reflection of a resting state, the fact that these were interspersed with activation tasks limits this interpretation. For example, since the controls had greater relative increase signal during the activation phase it is possible that this led to delayed increase in signal during baseline. Furthermore, the in-plane resolution used in this echo-planar study was $5.3 \mathrm{~mm}^{2}$, which prohibits complete subregional analysis. It remains unclear how age should influence resting signal measurements. We found no correlation between age and signal intensity; nevertheless, more data are needed to establish whether the age difference between the groups contributed to the effect. A general limitation of this data set is that regions other than the hippocampus were not evaluated, and the anatomical specificity of the finding cannot be addressed. These preliminary findings and their limitations were the basis for performing the second study.

\section{STUDY 2}

In this study, we used a gradient-echo pulse sequence to generate resting $\mathrm{T} 2 *$ maps of the hippocampal formation. This pulse sequence has been shown to be sensitive to changes in oxyhemoglobin/dexoyhemoglobin ratios (Frahm et al., 1993; Ogawa et al., 1990a), and its main advantage is that is provides enhanced spatial resolution compared to echo-planar imaging (Frahm et al., 1993).

\section{Subjects}

A new cohort of subjects were recruited from the two groups described in the first study: 7 patient's with probable $\mathrm{AD}$ (mean age $=76.8$, S.D. $=7.4$ ) and 6 healthy elderly with normal memory (mean age $=$ 79.5, S.D. = 5.1).

\section{Procedure}

As shown in Figure 2, a sagittal scout image was acquired to identify the long axis of the hippocampal formation. Five 5-mm oblique coronal slices were selected perpendicular to long axis of the hippocampal formation. The most anterior slice was always placed at the posterior aspect of the uncus, thus assuring the imaging of a slice appropriate for subregion localization (see below).

Foam pads and surgical tape were used to secure the head. Subjects were instructed to to close their eyes, and scanning was performed using a 1.5 tesla scanner. GRASS gradient-echo T2* -weighted images were acquired $(\mathrm{TE} / \mathrm{TR}=45 / 300$; flip angle $=10 ; \mathrm{NEX}=3$; $256 \times 256$ matrix; $24 \times 24 \mathrm{~cm}$ field of view; in-plane resolution $=0.9 \mathrm{~mm}^{2}$ ) The parameters of the pulse sequence were determined during preliminary studies. The T2* effect was optimized by titrating flip angle and TE time to the point where the lateral temporal was obscured by susceptibility artifact caused by the petrous bone (see Fig. 1). Signal to noise was improved by increasing NEX, and scan duration was minimized by manipulating TR and matrix size. The final scan time was 3:56 minutes.

Post-acquisition data processing was performed on a Silicon Graphics Indigo II work station, using image display and analysis software packages (IDL Research Systems). Data analysis was performed on a PC-based linux workstation using MEDx (Sensor systems), and was performed by an investigator blinded to subject grouping.

An approach used by animal investigators (Teyler, 1980) was adapted to identify the hippocampal subregions (as illustrated in Fig. 1):

- A single slice that provided optimal visualization of the required anatomical landmarks was selected. Importantly, this slice was never more than $20 \mathrm{~mm}$ 

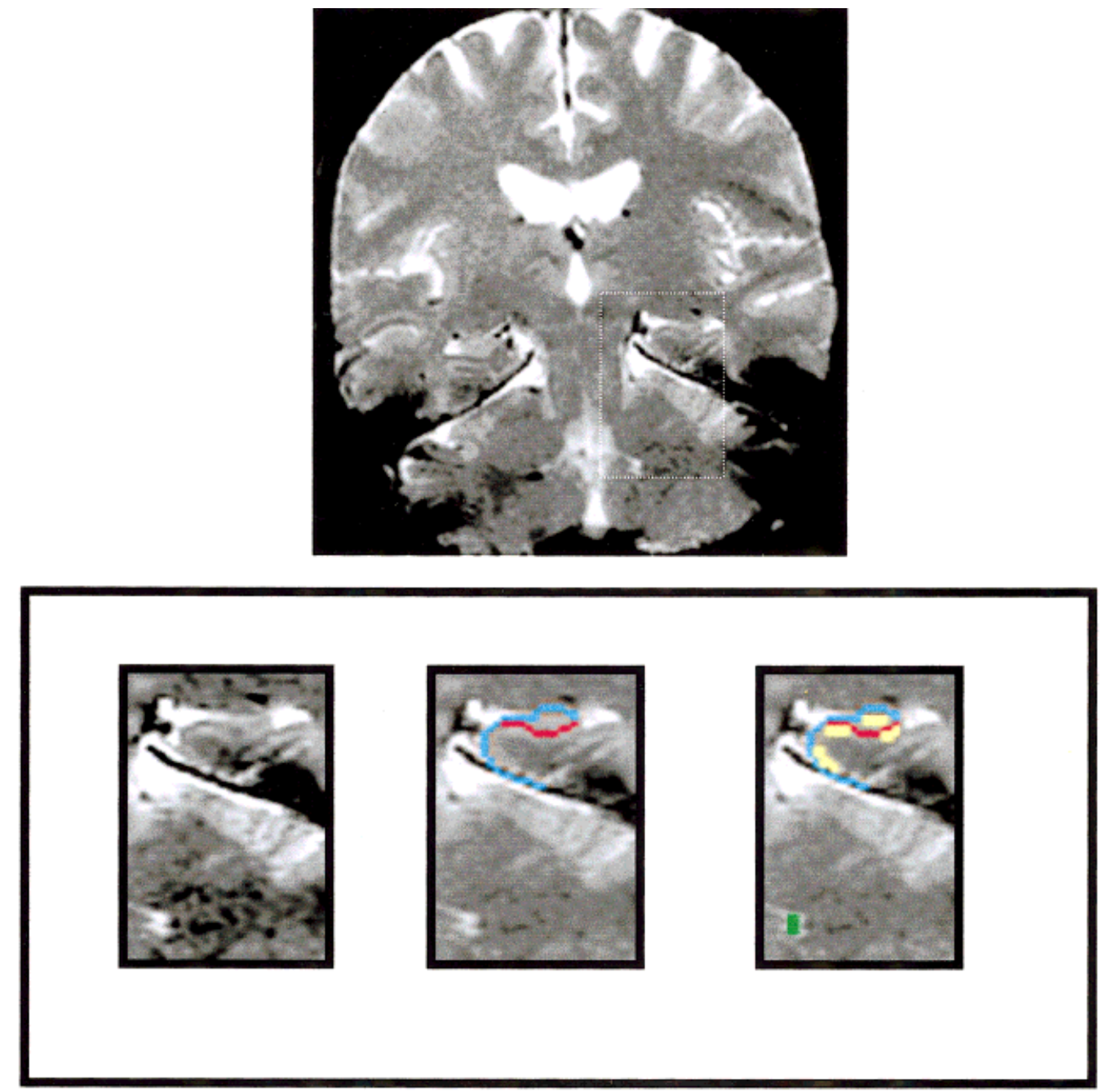

Fig. 2. Identifying the hippocampal subregions using high-resolution T2*-weighted images. Slice selection: The top panel is one of five transverse slices through the hippocampal formation. This slice was selected because it provided optimal visualization of the anatomical landmarks, and because it was anterior to the lateral geniculate nucleus. Anatomical landmarks: The external morphology of the hip-

(Amaral and Insausti, 1990) posterior to the amygdala, and was always anterior to the lateral geniculate body, assuring the slice contained the entorhinal cortex.

- The external morphology of the hippocampal formation was identified.

- The white matter tracts within the hippocampus were identified

- Pixels within the following four hippocampal subregions were selected for analysis: The entorhinal cortex, the subiculum, the CA1 subregion, and the dentate gyrus/CA3 subregion. Note that reliable landmarks distinguishing the dentate gyrus from the CA3 subfield could not be visualized, and they were combined.

\section{Data Analysis}

The average signal intensity of the pixels selected within each hippocampal subregion, for each subject, pocampus is identified (blue line). The internal white matter tracts are identified (red line). Pixel Selection: Pixels within the four hippocampal subregions are sampled (yellow). Border zones between the subregions are purposefully avoided. The dentate gyrus and the CA3 subfield are combined. Pixels within the dentate nucleus of the cerebellum (green) are sampled for comparison.

was determined. The average signal intensity of the dentate nucleus of the cerebellum was also determined. The dentate nucleus is not targeted by $\mathrm{AD}$ pathology and, therefore, provides an internal control. Global signal intensity can be influenced by potentially confounding variables such as day-to-day shifts in magnetic homogeneity. Furthermore, global T2*-weighted signals can be influenced by biological variables such as hematocrit and cardiac output. To control for these sources of inter-individual variability, global signal intensity was measured from the whole slice. The average signal intensity of each subregion was then divided by this global measure, yielding normalized signal intensities.

In order to test for group differences in resting T2*weighted signal, a multivariate analysis of variance (MANOVA) was performed. The between-subjects variable was group ( $\mathrm{AD}$ vs. healthy elderly), and the dependent variables were normalized signal intensity ac- 


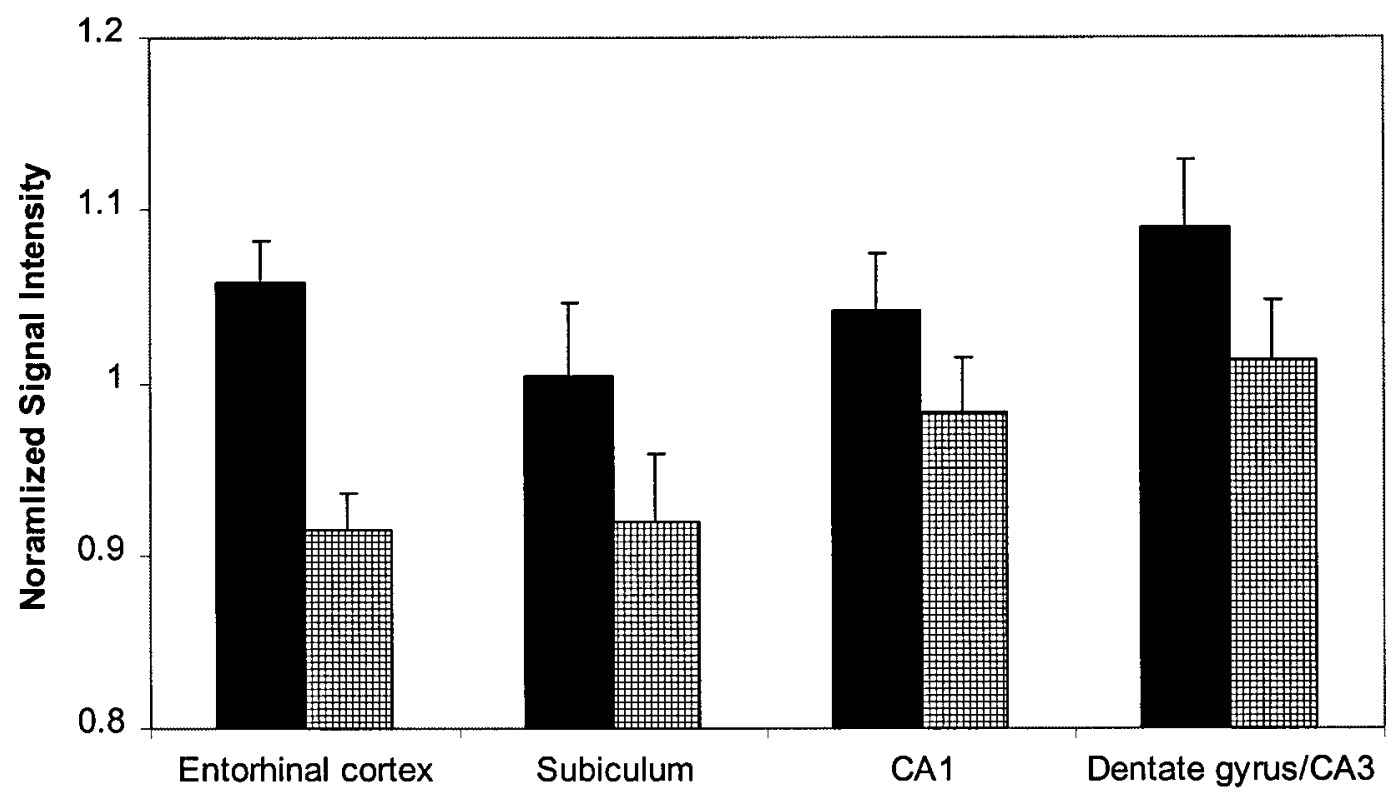

Fig. 3. Subregional analysis of the hippocampal formation comparing healthy elderly (solid bars) to patients with Alzheimer's disease (stippled bars). Signal intensity measured from each subregion normalized to global signal. Bars represent average group data with standard errors. Multivariate analysis found significant differences between Alzheimer's patients and healthy elderly. Univariate analysis found that the greatest difference was in the entorhinal cortex. quired in the entorhinal cortex, the subiculum, the CA1 subfield, and the combined dentate gyrus/CA3 subregion. An independent-sample $t$-test was performed to evaluate for group differences in normalized signal intensity of the dentate nucleus.

\section{Results}

As shown in Figure 3, the MANOVA revealed a significant difference between the groups in the normalized signal intensity, $\mathrm{F}(4,8)=7.2, P<.05$. Univariate analysis revealed the greatest difference in the entorhinal cortex, $\mathrm{F}(1,11)=20.7, P<.001$. The groups did not differ in the average number of pixels sampled from each subregion. The normalized average signal intensity of the dentate nucleus of the cerebellum was not significantly different between the two groups (healthy elderly mean $=1.23$, S.D. $=0.09$; Alzheimer's disease mean $=1.32$, S.D. $=0.19), \mathrm{t}(11)=-1.2, P>.10$.

\section{GENERAL DISCUSSION}

Using the relatively low-spatial resolution of echoplanar imaging, we first found that measurements of baseline $\mathrm{T} 2 *$-weighted signal from the hippocampal formation can dissociate AD from healthy elderly. We then used a protocol designed to perform subregional analysis of the hippocampal formation with superior spatial resolution (Small et al., submitted). This protocol dissociated AD from healthy elderly, with diminished resting signal found primarily in the entorhinal cortex.

This finding fits with the established pathological progression of $\mathrm{AD}$ pathology, where synaptic dysfunction and cell loss occurs first and foremost in the entorhinal cortex (Gomez-Isla et al., 1996; Masliah et al., 1994). Assuming that this difference in T2* -weighted signal is related to a static decrease in oxyhemoglobin/ deoxyhemoglobin ratio, should neuronal dysfunction be expected to result in this difference? It is reasonable to expect neuronal dysfunction to reduce local oxyhemoglobin delivery, but on theoretical grounds it is unclear what effect neuronal dysfunction would have on resting deoxyhemoglobin production. Plausible mechanisms can be invoked to predict an increase or a decrease in resting deoxyhemoglobin. A study using near infra-red spectroscopy, a method that can independently measure oxyhemoglobin and deoxyhemoglobin in living tissue, addressed this issue by comparing the frontal cortex of patients with $\mathrm{AD}$ to age-matched controls (Fallgatter et al., 1997). As expected, patients were found to have a significant decrease in resting oxyhemoglobin. Importantly, little change or even a slight increase was found in resting deoxyhemoglobin production. Thus, in keeping with the results from our study, $\mathrm{AD}$ pathology is expected to result in relative decrease in resting oxyhemoglobin/deoxyhemoglobin ratio.

In this study, only one clinical population was evaluated. Further validation of the protocol will be achieved by evaluating different clinical groups with suspected lesions to other hippocampal subregions.

How reliable is the approach used to identify the hippocampal subregions? All cases studied had a single slice that provided acceptable visualizations of the anatomical landmark used for subregion localization. Because of the small pixel dimensions, we were able to sample multiple pixels within a subregion, purposefully staying clear of the borderzones. Because the T2* -weighted maps provided sufficient anatomical resolution to identify the hippocampal subregions, there was no need for post-acquisition interpolation and co-registration onto a separately acquired "structural" MRI, 
which can diminish the fidelity of the signal source. Nevertheless, reliability of the approach will be best demonstrated by comparing the MR slice to post-mortem histological slices. We are planning on doing this with epilepsy patients with mesiotemporal sclerosis who undergo hippocampal transection for treatment.

The method has a number of limitations: (1) It is possible that certain pathologies result in physiological lesions that require direct activation in order to be detected. In this case dynamic, task-induced, fMRI would be more sensitive to these lesions. (2) Only single slices through the body of the hippocampus can be evaluated. This might not be appropriate for studies that need to evaluate the full longitudinal extent of the hippocampal formation. (3) Effects of some pathological processes-such as those resulting in gliosis or edema-would modify the signal independent of neuronal dysfunction. Furthermore, accumulation of iron (Thomas et al., 1993) or other metals might influence the signal. This could confound the results if accumulation occurred differently across experimental groups. (4) Although global signal intensity was used to normalize against sources of global signal variability, this does not address sources of local variability. For example, vasculopathy targeting an ateriole differentially feeding one subregion would modify $\mathrm{T} 2 *$ signal independent of neuronal dysfunction. Common causes of vasculopathy usually target larger-bore vessels upstream in the capillary tree, which in the hippocampus would influence perfusion to all subregions. Nevertheless, this confound remains a source of concern, as it is also for dynamic task-induced fMRI studies.

Other functional imaging techniques have relied on "resting" signal to detect brain dysfunction. The findings presented here are the first to show that a similar principle can be applied to MR measures of brain function in the hippocampal formation. Thus, the enhanced spatial resolution of MR technology can be exploited to evaluate dysfunction at the level of the hippocampal subregion. Since the subregion is the basic processing unit of the hippocampal circuit (McClelland et al., 1995), this level of analysis can provide greater insight into its normal and abnormal functional properties.

\section{REFERENCES}

Amaral DG, Insausti R. 1990. The Hippocampal Formation. In: Paxinos R, editor. The human nervous system. San Diego: Academic Press.

Amaral DG, Witter MP. 1989. The three-dimensional organization of the hippocampal formation: a review of anatomical data. Neuroscience 31:571-591.

Barnes CA. 1994. Normal aging: regionally specific changes in hippocampal synaptic transmission. Trends Neurosci 17:13-18.

Biswal B, Yetkin FZ, Haughton VM, Hyde JS. 1995. Functional connectivity in the motor cortex of resting human brain using echoplanar MRI. Magn Reson Med 34:537-541.

Bobinski M, de Leon MJ, Convit A, De Santi S, Wegiel J, Tarshish C Y, Saint Louis LA, Wisniewski HM. 1999. MRI of entorhinal cortex in mild Alzheimer's disease [letter]. Lancet 353:38-40.

Braak H, Braak E. 1996. Evolution of the neuropathology of Alzheimer's disease. Acta Neurol Scand Suppl 165:3-12.

Buschke H, Fuld PA. 1974. Evaluating storage, retention, and retrieval in disordered memory and learning. Neurology 24:10191025 .

Craik F, Anderson N, Kerr S, Li K. 1995. Memory changes in normal ageing. In: Baddeley A, Wilson B, Watts F editors. Handbook of memory disorders. West Sussex: John Wiley Sons Ltd. p 211-242. de la Torre JC, Fortin T, Park GA, Butler KS, Kozlowski P, Pappas BA, de Socarraz H, Saunders JK, Richard MT. 1992. Chronic cerebrovascular insufficiency induces dementia-like deficits in aged rats. Brain Res 582:186-95.

Duvernoy HM. 1998. The human hippocampus: an atlas of applied anatomy, 2nd ed..Munich: J.F. Bergman.

Fallgatter AJ, Roesler M, Sitzmann L, Heidrich A, Mueller TJ, Strik WK. 1997. Loss of functional hemispheric asymmetry in Alzheimer's dementia assessed with near-infrared spectroscopy. Brain Res Cogn Brain Res 6:67-72.

Forster MJ, Dubey A, Dawson KM, Stutts WA, Lal H, Sohal RS. 1996. Age-related losses of cognitive function and motor skills in mice are associated with oxidative protein damage in the brain. Proc Natl Acad Sci USA 93:4765-4769.

Frahm J, Merboldt KD, Hanicke W. 1993. Functional MRI of human brain activation at high spatial resolution. Magn Reson Med 29: 139-144.

Gabrieli JDE, Brewer JB, Desmond JE, Glover GH. 1997. Separate neural bases of two fundamental memory processes in the human medial temporal lobe. Science 276:264-266.

Galea LA, Perrot-Sinal TS, Kavaliers M, Ossenkopp KP. 1999. Relations of hippocampal volume and dentate gyrus width to gonadal hormone levels in male and female meadow voles. Brain Res 821:383-391.

Gazzaley AH, Siegel SJ, Kordower JH, Mufson EJ, Morrison JH. 1996. Circuit-specific alterations of N-methyl-D-aspartate receptor subunit 1 in the dentate gyrus of aged monkeys. Proc Natl Acad Sci USA 93:3121-3125.

Geinisman Y, Detoledo-Morrell L, Morrell F, Heller RE. 1995. Hippocampal markers of age-related memory dysfunction: behavioral electrophysiological and morphological perspectives. Prog Neurobiol 45:223-252.

Golomb J, Kluger A, de Leon MJ, Ferris SH, Convit A, Mittelman M S, Cohen J, Rusinek H, De Santi S, George AE. 1994. Hippocampal formation size in normal human aging: a correlate of delayed secondary memory performance. Learn Mem 1:45-54.

Gomez-Isla T, Price JL, McKeel DW Jr, Morris JC, Growdon JH, Hyman BT. 1996. Profound loss of layer II entorhinal cortex neurons occurs in very mild Alzheimer's disease. J Neurosci 16:44914500 .

Helkala EL, Koivisto K, Hanninen T, Vanhanen M, Kervinen K, Kuusisto J, Mykkanen L, Kesaniemi Y A, Laakso M, Riekkinen P Sr. 1995. The association of apolipoprotein E polymorphism with memory: a population based study. Neurosci Lett 191:141-144.

Jacobs DM, Sano M, Dooneief G, Marder K, Bell KL, Stern Y. 1995. Neuropsychological detection and characterization of preclinical Alzheimer's disease [comment] [see comments]. Neurology 45: 957-962.

Masliah E, Mallory M, Hansen L, DeTeresa R, Alford M, Terry R. 1994. Synaptic and neuritic alterations during the progression of Alzheimer's disease. Neurosci Lett 174:67-72.

McClelland JL, McNaughton BL, O'Reilly RC. 1995. Why there are complementary learning systems in the hippocampus and neocortex: insights from the successes and failures of connectionist models of learning and memory. Psychol Rev 102:419-457.

McEwen BS. 1994. Corticosteroids and hippocampal plasticity. Ann N Y Acad Sci 746:134-142; discussion 142-144, 178-179.

McKhann G, Drachman D, Folstein M, Katzman R, Price D, Stadlan EM. 1984. Clinical diagnosis of Alzheimer's disease: report of the NINCDS-ADRDA Work Group under the auspices of Department of Health and Human Services Task Force on Alzheimer's Disease. Neurology 34:939-944.

Morris JC. 1993. The Clinical Dementia Rating (CDR): current version and scoring rules [see comments]. Neurology 43:2412-2414

Nicoll RA, Malenka RC. 1995. Contrasting properties of two forms of long-term potentiation in the hippocampus. Nature 377:115-118.

Ogawa S, Lee TM, Kay AR, Tank DW. 1990a. Brain magnetic resonance imaging with contrast dependent on blood oxygenation. Proc Natl Acad Sci USA 87:9868-9872.

Ogawa S, Lee TM, Nayak AS, Glynn P. 1990b. Oxygenation-sensitive contrast in magnetic resonance image of rodent brain at high magnetic fields. Magn Reson Med 14:68-78.

Petersen RC, Smith G, Kokmen E, Ivnik RJ, Tangalos EG. 1992. Memory function in normal aging. Neurology 42:396-401.

Simic G, Kostovic I, Winblad B, Bogdanovic N. 1997. Volume and number of neurons of the human hippocampal formation in normal aging and Alzheimer's disease. J Comp Neurol 379:482-494.

Small SA, Perera GM, DeLaPaz R, Mayeux R, Stern Y. 1999a. Differential regional dysfunction of the hippocampal formation among elderly with memory decline and Alzheimer's disease. Ann Neurol $45: 466-472$. 
Small SA, Stern Y, Tang M, Mayeux R. 1999b. Selective decline in memory function among healthy elderly. Neurology 52:1392-1396. Stern CE, Hasselmo ME. 1999. Bridging the gap: integrating cellular and functional magnetic resonance imaging studies of the hippocampus. Hippocampus 9:45-53.

Stern Y, Andrews H, Pittman J, Sano M, Tatemichi T, Lantigua R, Mayeux R. 1992. Diagnosis of dementia in a heterogeneous population. Development of a neuropsychological paradigm-based diagnosis of dementia and quantified correction for the effects of education. Arch Neurol 49:453-460.

Teyler TJ. 1980. Brain slice preparation: hippocampus. Brain Res Bull 5:391-403.
Thomas LO, Boyko OB, Anthony DC, Burger PC. 1993. MR detection of brain iron [see comments]. AJNR Am J Neuroradiol 14:10431048.

West MJ. 1993. Regionally specific loss of neurons in the aging human hippocampus. Neurobiol Aging 14:287-293.

Woods RP, Mazziotta JC, Cherry SR. 1993. MRI-PET registration with automated algorithm. J Comput Assist Tomogr 17:536-546.

Xiong J, Parsons LM, Gao JH, Fox PT. 1999. Interregional connectivity to primary motor cortex revealed using MRI resting state images. Hum Brain Map 8:151-156.

Zola-Morgan S, Squire LR. 1993. Neuroanatomy of memory. Annu Rev Neurosci 16:547-563. 